\title{
EL REFLEJO DE LA GESTIÓN DEL RIESGO EN LAS PÁGINAS DE LA REVISTA GEOLÓGICA DE AMÉRICA CENTRAL
}

\author{
RISK MANAGEMENT REFLECTED IN THE PAGES OF \\ THE CENTRAL AMERICAN JOURNAL OF GEOLOGY
}

\author{
Giovanni Peraldo ${ }^{1 *} \&$ Benjamín Acevedo ${ }^{2}$ \\ ${ }^{1}$ Escuela Centroamericana de Geología, Universidad de Costa Rica \\ ${ }^{2}$ Escuela de Antropología, Universidad de Costa Rica \\ *Autor para contacto: gperaldoh@gmail.com
}

(Recibido: 11/08/2014; aceptado: 09/09/2014)

\begin{abstract}
As part of the celebration of the thirtieth anniversary of the Geological Journal of Central America, an analysis of the issue of disasters is currently studied in the field of risk management and how this issue is addressed in the content of the articles published in the magazine. Are chosen for analysis articles explicitly refer to issues related to risk management, it is obvious that the largest number of items RGAC relate to the issue of risk, they address relevant aspects for management as is the characterization of the processes that generate threats (tectonics, seismology, volcanology, geotechnical), among others. Risk is an intrinsic characteristic of the transformation of the environment, both the quantitative and qualitative study of the transformation inputs conducive to the prevention and land use planning. The paradigm shift from the study of the event as synonymous with the paradigm of disaster risk management gives importance to the construction of risk scenarios on the prediction and prognosis related to tectonic events. Keywords: Risk management, threat, vulnerability, risk, land planning.

RESUMEN: Como parte de la celebración de los treinta años de la Revista Geológica de América Central, se hace un análisis sobre el tema de los desastres, actualmente estudiado en el campo de la Gestión del Riesgo y cómo se aborda dicho tema en los contenidos de los artículos publicados en la revista. Se escogen para su análisis los artículos que de manera explícita hacen referencia a temas relacionados con la gestión del riesgo, pues es obvio que la mayor cantidad de artículos de la RGAC tienen relación con el tema del riesgo, pues abordan aspectos relevantes para su gestión como es la caracterización de los procesos generadores de amenazas (tectónica, la sismología, vulcanología, la geotecnia), entre otros. El riesgo es una característica intrínseca de la transformación del ambiente, por tanto el estudio cuantitativo y cualitativo de dicha transformación propicia insumos para la prevención y el ordenamiento territorial.

El cambio de paradigma desde el estudio del evento como sinónimo de desastre al paradigma de la gestión del riesgo da importancia a la construcción de escenarios de riesgo por sobre la predicción y el pronóstico para eventos relacionados con la tectónica.
\end{abstract}

Palabras clave: Gestión del riesgo, amenazas, vulnerabilidad, riesgo, ordenamiento territorial. 


\section{INTRODUCCIÓN}

En este año 2014 la Revista Geológica de América Central (RGAC), cumple tres décadas de continua divulgación de investigaciones en ciencias geológicas de la región centroamericana y del Caribe. Esto hace que se deba hacer un atisbo retrospectivo hacia los campos de la geología representados en este medio de difusión científica, para otear las preferencias que históricamente han sido temas de interés e identificar los avances en esos campos. Además nos remite a la dirección que se ha dado a la enseñanza de la geología y las exigencias que hoy tiene la sociedad hacia la aplicación de las ciencias geológicas. Es dentro de esta línea de pensamiento, que se presenta este artículo que recorre el tema de los desastres, actualmente estudiados en el campo conocido como gestión del riesgo y observar su construcción a lo largo de seis lustros de investigación.

La escogencia de los artículos y notas técnicas que serán comentados en este artículo es difícil por cuanto muchos de los resultados de investigación publicados en la RGAC muestran una relación implícita con la gestión del riesgo, pues son estudios de procesos o de eventos que aportan valiosa información hacia otros campos relacionados con el triduo ambiente - desarrollo - riesgo. Se decide abordar solamente aquellos cuya relación con el tema de la gestión del riesgo se da de manera explícita sea en el título o bien en las palabras clave. Se agrupan los artículos y notas técnicas en dos momentos concretos de la revista: 1984 a 2003 periodo sin palabras clave en los artículos y de 2003 al presente, periodo en el que se empieza a incorporar las palabras clave que reflejan el temario abordado en un determinado artículo.

Pero también esos períodos coinciden, en parte, con la evolución del paradigma sobre el tema del desastre: a partir de la idea de desastre natural y el proceso del desastre como un ciclo se llega al tema integral e interdisciplinario de la gestión del riesgo. El primer periodo se trabajó según el título del artículo y su resumen, y el segundo periodo se analizó según palabras clave más resumen, esto con el fin de identificar temáticas y autores.

\section{GESTIÓN DEL RIESGO}

El análisis de las investigaciones de lo publicado en la RGAC sobre gestión del riesgo es complejo, por cuanto el tema está aún en construcción, el cuerpo teórico en que se fundamenta ha evolucionado así como su objeto de estudio. Inicialmente el tema se redujo al estudio del concepto "desastre natural" tal como queda consignado en los primeros artículos y notas técnicas publicadas en la revista, tales como en Paniagua (1986). Vergara (1987) piensa el término desastre natural como sinónimo del evento geológico, lo que a todas luces actualmente se asocia al concepto de amenaza. Según Vergara (1987) el punto de interés es el evento natural fortuito, siendo así el único aspecto a considerar en una investigación para reducir las pérdidas, pues aunque define vulnerabilidad y riesgo específico, la sociedad es observada como un elemento pasivo, víctima de la naturaleza.

Desde la visión de desastre natural, el evento no podía ser prevenido o mitigado, pues no se pueden identificar todos los factores disparadores de la manifestación natural súbita (p.e. un sismo o una erupción). El concepto estaba enfocado hacia el estudio del proceso natural y el término "desastre" fue directamente referido a la destrucción generada por el evento natural, por lo tanto se entendía como un sinónimo del evento (terremoto, erupción, deslizamiento, inundación, entre otros). Desde un enfoque fisicalista, la ocurrencia del "desastre natural" se ve como una posibilidad de recolectar sendos datos en laboratorios especializados y su posterior análisis, con el fin de interpretar el origen del evento, sus parámetros físicos característicos y conocer la estructura geológica de una región geográfica determinada.

Un modelo que fue explotado en la década de 1990 con el fin de integrar desastre y desarrollo se llamó el Ciclo de los Desastres que es presentado en una nota técnica por Paniagua (1995). Dicho modelo concibe los desastres como un ciclo, en el cual la sociedad queda inmersa en la eterna lucha entre el desastre y el proceso de desarrollo. Es una repetición exacta de etapas en el siguiente orden de aparición y de ejecución: evento - respuesta - 
rehabilitación - reconstrucción - prevención - mitigación - preparación - alerta - evento (se completa el ciclo) (Paniagua, 1995). Sin embargo, el ciclo no sobrevive a un breve análisis en cuanto a sus etapas, pues al basarse en una alerta, entonces el modelo queda obsoleto para eventos provenientes de procesos asociados a la geodinámica interna. Además, al quedar la prevención como una etapa dentro del ciclo, el modelo vuelve a quedar obsoleto, por cuanto prevención no puede ser entendido como una etapa sino como un tema que debe ser transversal al proceso de desarrollo. El modelo no sale del esquema de respuesta de intervención inmediatamente después del impacto del evento natural.

Una herencia de tal paradigma son los estudios dirigidos a la predicción, y la millonaria transferencia de tecnología, que de alguna manera responde a los modelos económicos capitalistas de las últimas décadas del siglo XX. Al ser el evento natural el causante de los daños y muertes, la sociedad está completamente indefensa ante tales acontecimientos de manifestación súbita, entonces, la protección de la sociedad ante los "avatares del destino", exige conocer el momento en que un terremoto o una erupción ocurrirá, de acá nace la idea de predecir el evento natural súbito, para alertar a las poblaciones que pueden ser impactadas por tal evento. Sin embargo, mientras los científicos insistían en afinar los modelos y métodos de predicción, el desarrollo socioeconómico en las áreas de intensa actividad continuaba ajeno a los procesos naturales del área en desarrollo. Pero además y según el enfoque de "desastre natural", la destrucción que un sismo o una erupción pudieran provocar en la sociedad impactada, ha sido usada de manera mediática para justificar acciones religiosas o condenar proyectos políticos, empleando para ello el miedo (Peraldo \& Mora, 2009; Peraldo \& Mora, 2010; Peraldo \& Acevedo, 2014).

Sin embargo, el tratamiento del concepto "desastre natural" empezó a generar cuestionamientos desde otras áreas del saber científico, tales como las Ciencias Sociales. Sendas colecciones de artículos filosóficos empezaron a circular dentro de la comunidad científica preocupada por el tema. Así se reconoce el esfuerzo de la Red de Estudios
Sociales en Prevención de Desastres en América Latina, que desde mediados de la década de 1980 comenzó un bombardeo de ideas que ya venían manifestando autores como Enrique Florescano, Allan Labell, Andrew Maskrey, Virginia García Acosta, Gustavo Wilchez Chaux, Allain Musset, entre otros. En palabras sencillas, según el nuevo enfoque la sociedad no puede interpretarse como un elemento pasivo, sino como una fuente de vulnerabilidades y de riesgos que amplifican las consecuencias derivadas de un evento natural. Además ese nuevo enfoque involucra la teoría de sistemas, el ambiente, el desarrollo como partícipes. El evento natural queda como una parte integral del estudio del riesgo. Es la antesala de la declaratoria de los años de 1990 como el Decenio de los Desastres Naturales, propuesto por la ONU, ante el hecho de ver en los desastre un escollo para el desarrollo, según lo señala Paniagua (1995).

Desde un enfoque geográfico y sociológico, la sociedad no es un fenómeno estático pasivo dentro de un territorio determinado, sino el resultado de un complejo proceso, que conforma un sistema abierto donde se intercambia materia y energía entre territorios definidos. El enfoque que empezaba a transformar el concepto de desastre natural, involucró cada vez con más intensidad a la sociedad y sus características. Para entender este enfoque es importante señalar acá lo que la academia de la Lengua Española define por territorio como una "Porción de la superficie terrestre perteneciente a una nación, región, provincia...".

Obsérvese que tanto espacio como sociedad son partes indivisibles del concepto territorio. Este está formado por uno o más paisajes. Bolós (1992), sustenta las transformaciones de paisajes -y por tanto de los territorios- como consecuencia de un intercambio de materia y energía. Siguiendo esta idea Peraldo (2004) la aplica para explicar el proceso de desastre, tal como un flujo bidireccional de masa y energía, entre porciones de un territorio o entre territorios y que generan desequilibrios importantes por efectos de un conjunto de vulnerabilidades. Esta idea se refuerza con el planteamiento de Santos (1990), quien propone que las relaciones que ocurren en el espacio permiten que éste 
se estructure y evolucione. ¿Será posible que los desastres se entiendan como una manifestación de esa estructuración y evolución?

El tipo de relaciones que ocurren en el espacio son las ambientales y el espacio es el contenedor de ellas. Ambiente podría ser entendido como las relaciones biunívocas entre lo biótico y lo inorgánico que tiene lugar en un territorio determinado.

El territorio es susceptible de ordenarse política y espacialmente en función de las características naturales y de la capacidad de uso de todas sus partes integrantes. Por tanto el ordenamiento es parte esencial de la gestión, que implica administrar el territorio con el fin de prever y mitigar resultados perniciosos a raíz de los diversos procesos que tienen lugar en el ambiente respectivo.

Entonces, en la relación sociedad - espacio se pueden plantear propuestas socioeconómicas deficitarias y desligadas de los procesos geológicos y climáticos, en ausencia de políticas atinadas de desarrollo en la justa medida con las características naturales del territorio donde esas propuestas han de ser aplicadas, pueden generar una serie de inconsistencias en el proceso de desarrollo. En otras palabras, las políticas tendientes a impulsar una planificación estratégica socioeconómica en desequilibrio con el medio adolecen la ausencia de prevención.

Se considera en este artículo que las inconsistencias anteriormente citadas vienen a ser identificadas con la vulnerabilidad, entendida grosso modo como el grado de exposición de un elemento social o estructura física, a una determinada amenaza. La vulnerabilidad no es constante en el espacio para una amenaza específica, pues depende de las características y distancia del elemento vulnerable a la fuente de amenaza, y socialmente varía en función de edad, género, cultura o ingresos económicos. Se analiza junto con las capacidades, esto, porque si solamente se observan las vulnerabilidades, estamos restringiendo la sociedad a una visión negativa en su construcción y desarrollo. La sociedad también tiene capacidades para disminuir el peligro que representa la exposición ante determinada amenaza, implica que con este enfoque se estudian de manera integral las amenazas, las vulnerabilidades y con esos dos parámetros se calcula el riesgo. Según todo esto ya no interesa, por ejemplo, cuándo ocurrirá un sismo, interesa cuán vulnerable está un grupo humano para resistirlo y por tanto, cuán versátil es en función de su resiliencia, interesa conocer su escenario de riesgo y cómo éste evoluciona con el fin de atacar las vulnerabilidades.

Por tanto, los estudios del proceso natural o de un evento natural específico se deben conjugar con los análisis de la sociedad y sus procesos, con el fin de que se pueda reducir satisfactoriamente el riesgo. Se complica más su estudio, porque al evolucionar la sociedad y por tanto el territorio, se transforman las relaciones que Santos (1990) identifica como causantes para que el espacio constantemente se estructure y evolucione, lo que genera nuevas vulnerabilidades y riesgos. En otras palabras los escenarios de riesgo están en constante evolución. Esto significa que los planes reguladores cantonales que controlan el desarrollo de una sociedad en un territorio dado, deben ser revisados constantemente, por cuanto se desactualizan por su función en sí mismos.

Como ya se puede suponer, los conceptos vulnerabilidad y riesgo han generado una serie de replanteamientos en cuanto a las bases teóricas y metodológicas del estudio de los desastres. Actualmente la gestión del riesgo es un tema envolvente de muchos otros que estudian, desde lo natural hasta lo social, la construcción de escenarios de riesgo. Es un tema de estudio de la geología ambiental, pues aporta la teoría y el método para su investigación, un ejemplo es la creación de mejores modelos para disminuir la degradación ambiental y entender los procesos naturales y antrópicos amenazantes del territorio (ver Servicio Geológico Mexicano, 2014).

Por lo tanto existe un vínculo entre la gestión del riesgo, el ordenamiento territorial y la gestión ambiental, esto permite que el proceso de desarrollo de una sociedad se mueva entre los extremos de la seguridad y el riesgo (Fig. 1).

La gestión del riesgo busca administrar el riesgo que puede generarse en las relaciones ambientales primarias y secundarias, con el fin de que el riesgo no sea un obstáculo al proceso de 


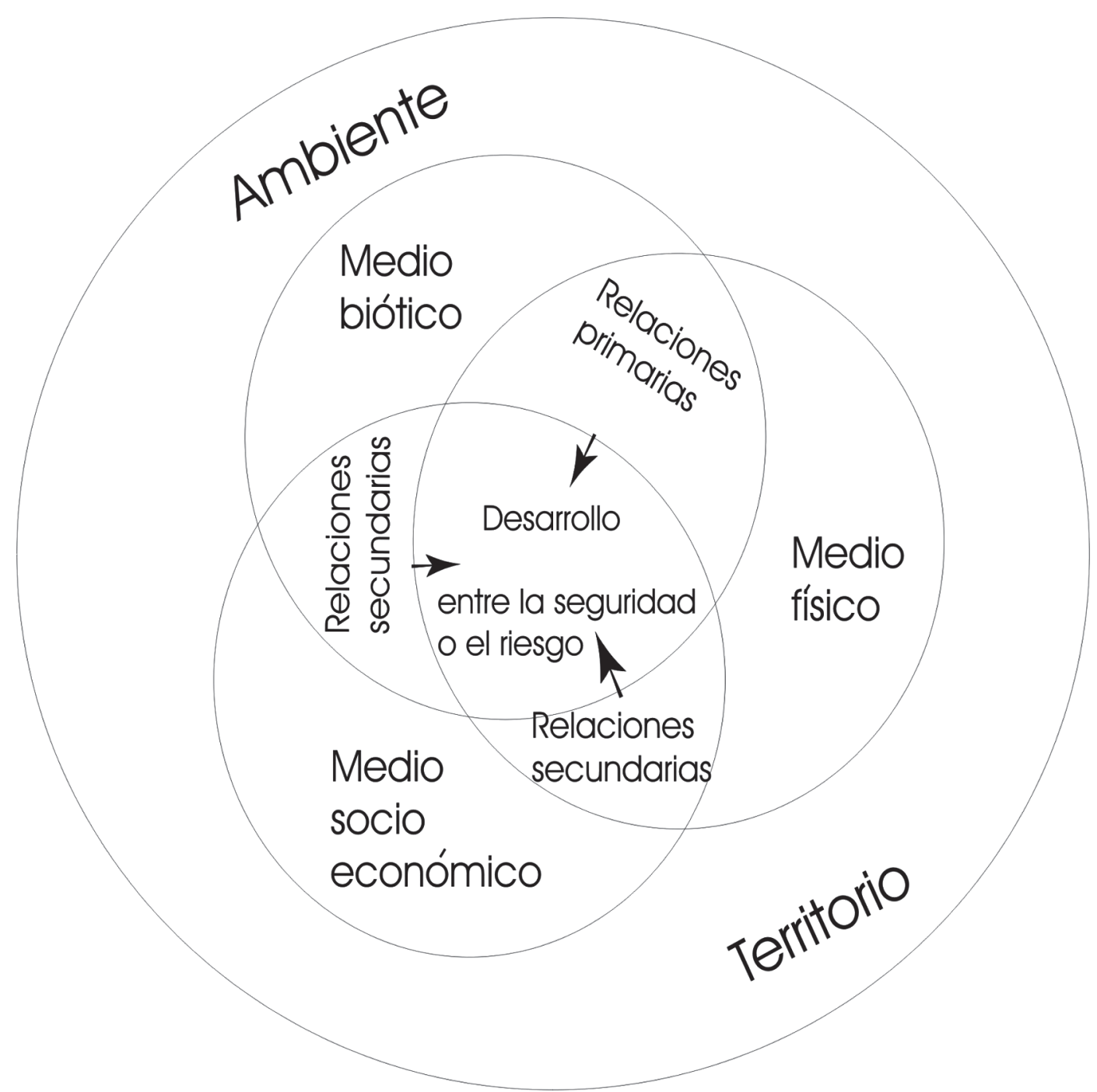

Fig. 1: Relaciones entre el ambiente, el territorio y el desarrollo, este último se mueve entre los extremos de seguridad y riesgo, dependiendo de que tanto se adapte a las relaciones ambientales primarias y secundarias.

desarrollo. También estudia la evolución del riesgo, de tal manera que se identifiquen los escenarios de riesgo y su evolución y se mantengan en límites no peligrosos, y para lograrlo se requiere identificar las amenazas existentes, tanto geológicas como antrópicas, identificar la vulnerabilidad, generar zonificaciones con el fin de contribuir con el ordenamiento territorial, el cual se supone, organiza el desarrollo en una región para lo cual toma en cuenta sus características ambientales pero todo lo anterior dentro de un pensamiento de prevención (Fig. 2). ¿Se hablará de lo mismo cuando se separan las diversas gestiones del riesgo, del ambiente o del territorio?

Diversos escenarios de riesgo coexisten en áreas urbanas. La Fig. 2A muestra contrastes en el desarrollo de ciudades como Caracas, que al igual que Tegucigalpa (Fig. 2D) coexisten barrios marginales sujetos a inestabilidad de laderas y áreas económicamente más desarrolladas. El barrio de la 

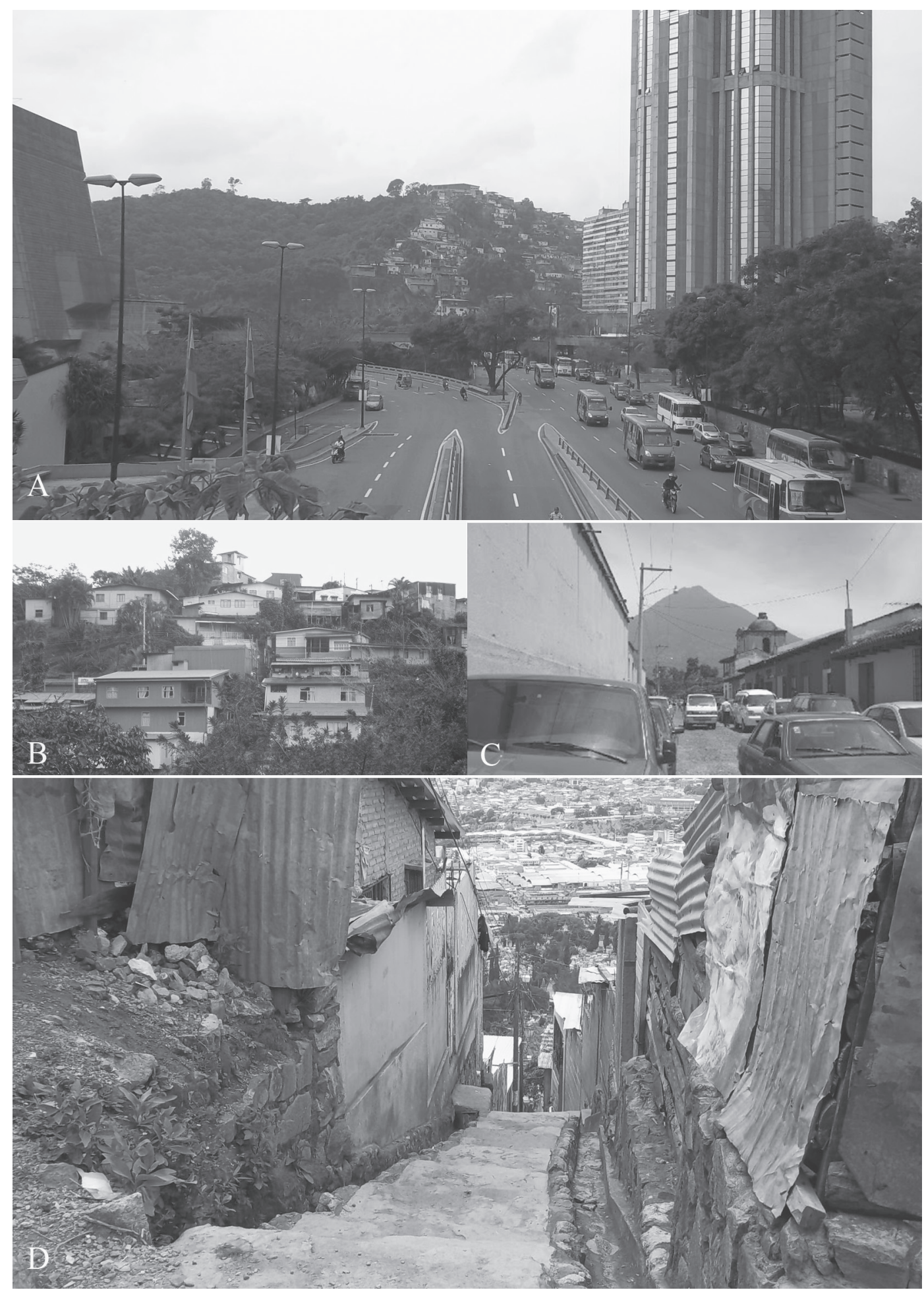
Fig. 2D se denomina Campo Cielo y persiste a la par del deslizamiento El Berrinche activo durante el impacto del Huracán Mitch en 1998. Campo Cielo está afectado por un sector del citado deslizamiento, siendo así que han ocurrido agrietamientos y movimientos de tierra importantes que han afectado viviendas. Obsérvese la pendiente y los pasillos estrechos propios de poblaciones hacinadas. Antigua Guatemala (Fig. 2C) es una ciudad que a lo largo de su historia ha sido sumamente afectada por sismos, al punto de perder su estatus de capital de la Capitanía de Guatemala después de los terremotos de Santa Marta de julio de 1773 (Peraldo, 2014). En Costa Rica las relaciones ambientales y el urbanismo no nos depara nada nuevo, pues la Fig. 2B muestra viviendas (Grecia- Sarchí) de clase media colgando de pendientes fuertes, cuya litología corresponde con tobas fuertemente meteorizadas. Ya en las cercanías de dicha localidad el sismo del 5 de septiembre de 2012 afectó fuertemente viviendas ubicadas en sectores con características similares.

La relación entre la gestión del riesgo y la gestión ambiental es clara cuando se analiza en toda su magnitud la ocurrencia de un desastre, que es lo mismo que la actualización del riesgo, a partir de escenarios de vulnerabilidad tal como los anotados en la Fig. 2 y diagramados en la Fig. 3.

El análisis de las características ambientales de un sector, es una valiosa información para ser aplicada a la gestión del riesgo. La Fig. 4 muestra dos ejemplos concretos de características ambientales adversas para el desarrollo urbano.

La Fig. 4A muestra una vivienda ubicada entre El Descanso y Pacayas, Cartago, Costa Rica (coordenadas 558,30-209,08) destruida en 1997 por el desbordamiento de la quebrada El Presídio durante una fuerte precipitación. Pese que la vivienda se encontraba cerca de 300 metros del puente de esa quebrada, y cerca de 500 metros al sur del punto donde la quebrada se desbordó, el factor morfológico característico del campo de lavas de Cervantes, en donde se pueden encontrar colinas alargadas y depresiones entre coladas, permitió que el agua y lodo del desbordamiento se canalizó por las leves depresiones de las laderas y llegó con mucha energía a golpear, levantar desde los cimientos y depositar sobre la carretera la vivienda. Este es un ejemplo de cómo las características ambientales se conjugan para generar amenazas localizadas a mucha distancia del factor de riesgo y que sin un análisis adecuado se le asignaría una baja o inexistente vulnerabilidad por distancia del factor de riesgo. En 4B se observa una vivienda destruida por la reactivación de un deslizamiento rotacional a raíz de las precipitaciones generadas por el Huracán Mitch en octubre de 1998. Este sector se localiza en San Juan de Limay, departamento de Estelí, Nicaragua. Sin embargo, durante la fundación de la población ese factor de riesgo no había sido identificado y el pueblo creció ignorando la susceptibilidad al deslizamiento. El problema radica en que posterior a la detección de tal proceso se continúe con el desarrollo socioeconómico en el área.

De todos modos, existen muchas limitantes que hacen imposible hacer efectivos los objetivos de la gestión del riesgo y ambiental que cristalice en un proceso de ordenamiento territorial. Entre esas limitantes pueden citarse: (1) la conceptualización occidental acerca de la naturaleza, (2) la historia del desarrollo de centros urbanos, (3) el quehacer del aparato estatal descaminado de un proceso de prevención, (4) la gestión del riesgo se tornó en un vehículo mediático, en donde la producción de teoría supera con creces su aplicación concreta, (5) el alejamiento de las políticas económicas de las sociales; (6) la incapacidad del aparato estatal para hacer que los procesos de desarrollo sean sostenibles y sustentables, entre otras limitantes que literalmente

Fig. 2: Áreas urbanas con contrastes de desarrollo. A. Caracas, edificios y vías que denotan un gran desarrollo pero con población vulnerable en áreas de inestabilidad de laderas, foto G. Peraldo, 27 -10-2012. B. Viviendas de clase media ubicadas en fuerte ladera, entre Grecia y Sarchí, foto G. Peraldo, 12-11-2012. C. Santiago de los Caballeros (Antigua Guatemala), al fondo el volcán de Agua y vías estrechas y congestionadas en una ciudad sometida a terremotos. Foto G. Peraldo, marzo de 2006. D. Campo Cielo, Tegucigalpa, caserío pauperizado, amenazado por la reactivación de un sector lateral del deslizamiento El Berrinche. Foto G. Peraldo, julio de 2011, Campaña Geológica. 


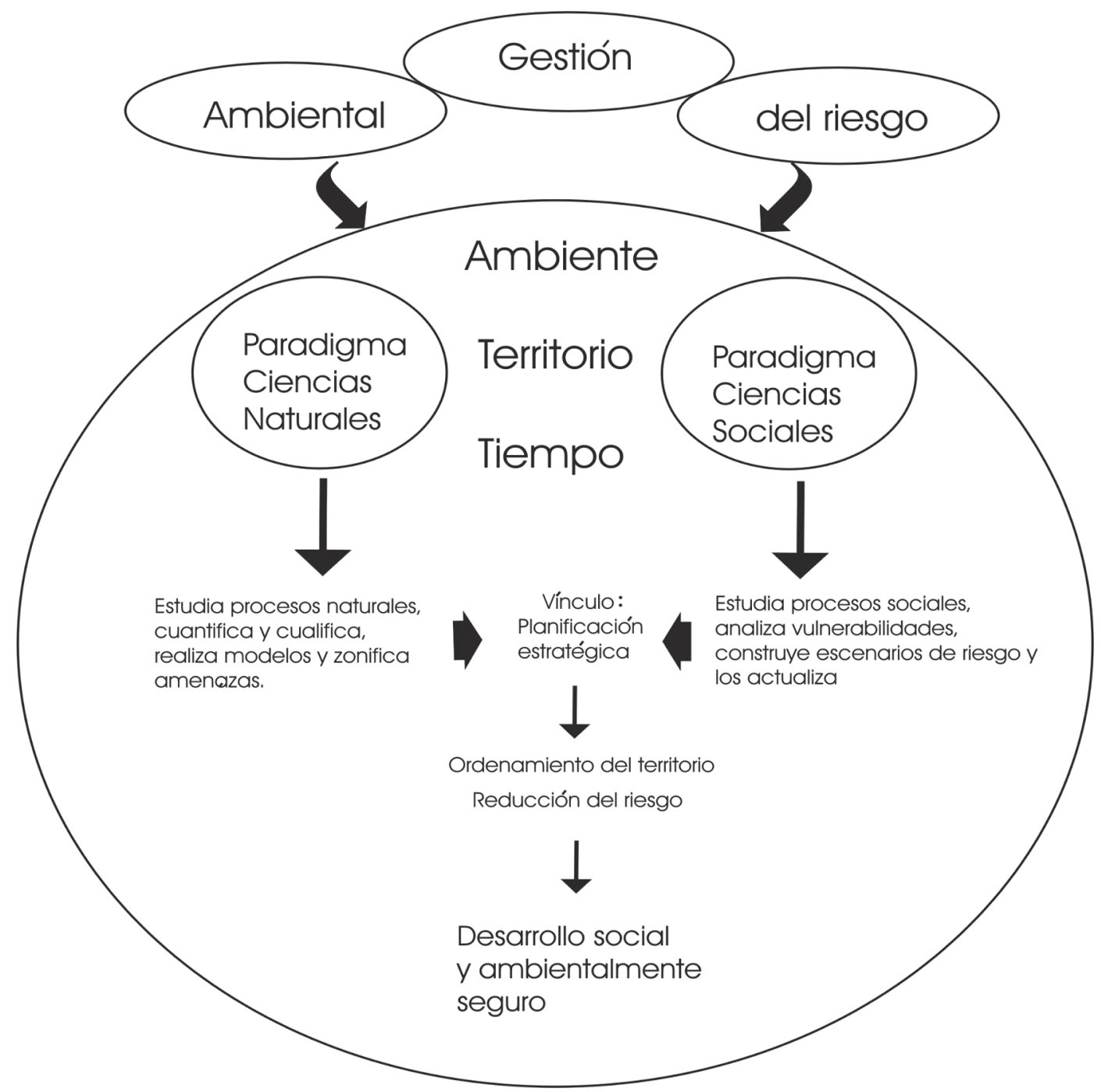

Fig. 3: Estudio integral del ambiente y el territorio desde la gestión del ambiente y del riesgo, en donde el análisis será enfocado desde dos paradigmas: ciencia natural o “dura” y ciencia social “¿suave?”. El vínculo entre ellos será la planificación estratégica del territorio que permite crear un desarrollo social y ambientalmente seguro.

hacen que la gestión del riesgo, del ambiente y el ordenamiento territorial existan en la literatura especializada pero no en la realidad concreta.

\section{HISTORIAL DE ARTÍCULOS}

Los artículos y notas técnicas sobre el tema de gestión del riesgo que ha publicado la RGAC que se discutirán a continuación de manera sucinta son los que se refieren explícitamente en el título o en las palabras clave a la temática de gestión del riesgo. Esta discusión intenta observar cómo se refleja la evolución conceptual sobre el tema.

También es claro que la RGAC muestra una buena cantidad de artículos directamente relacionados con la gestión del riesgo. Por ejemplo, los artículos que versan sobre el análisis de sistemas de fallamiento, de procesos magmáticos en volcanes activos, estudio de eventos, o bien el análi- 


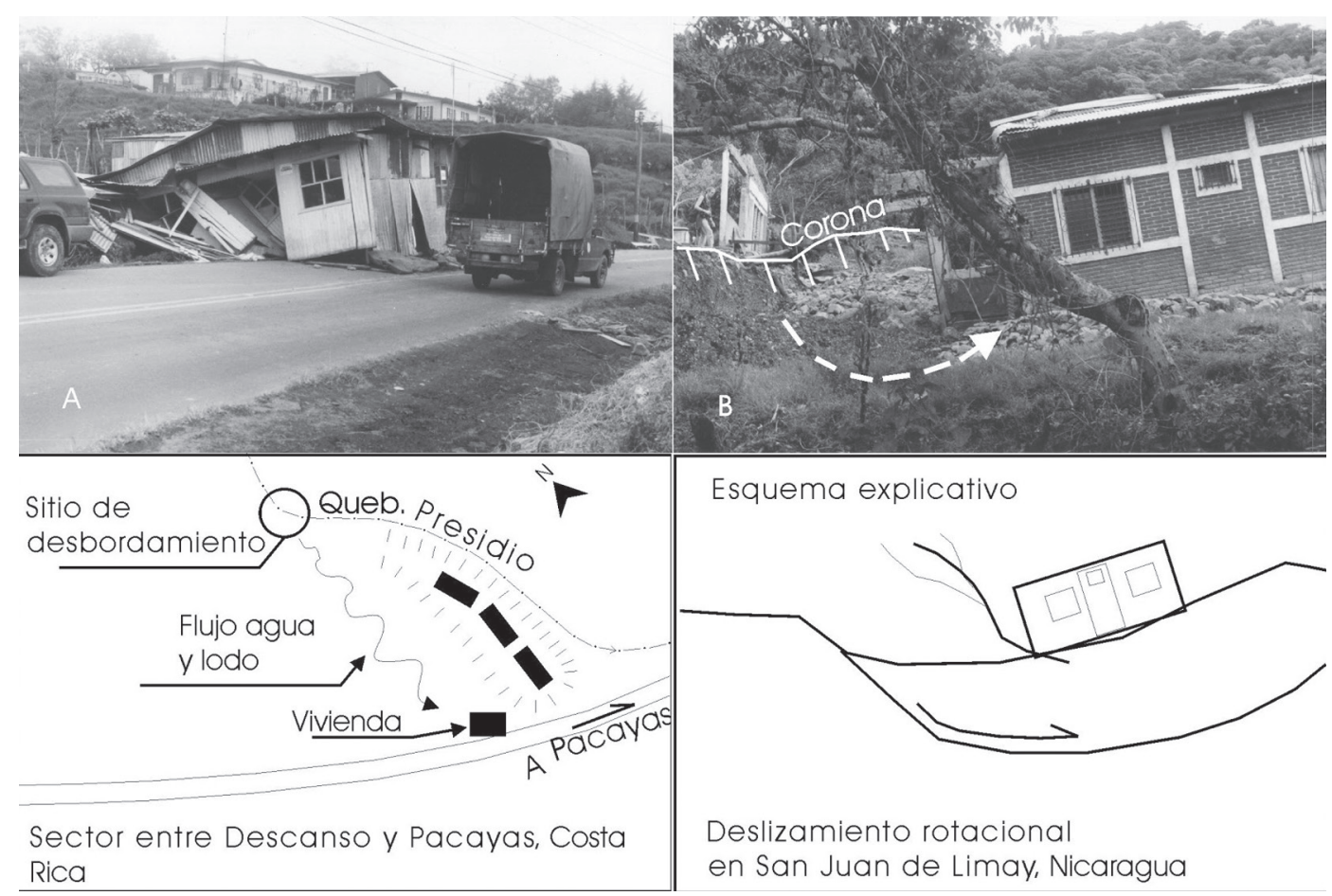

Fig. 4: A. Casa afectada en 1997 por desbordamiento de la Quebrada El Presídio en coordenadas 558,30-209,08. Los rectángulos negros de la derecha corresponden con las viviendas ubicadas sobre una colina alargada y que se observan detrás de la vivienda afectada. La Quebrada El Presídio tiene su cauce detrás de la colina. En el esquema sin escala, el sitio del desbordamiento se encuentra aproximadamente $500 \mathrm{~m}$ de distancia de la vivienda afectada. Se muestra en la fotografía el esquema para entender el problema. Foto, G. Peraldo. B. Vivienda destruida e inclinada por un deslizamiento rotacional ubicado en el Departamento de Matagalpa, Nicaragua, durante el huracán Mitch. Foto G. Peraldo.

sis geotécnico de un tipo de suelo específico, son investigaciones básicas para establecer necesidades de prevención. No obstante, no se observa el vínculo, a veces muy fino, entre la investigación científica pura y su aplicación a la sociedad. Acá es importante, por tanto, hacer la diferencia entre los conceptos de proceso geológico, su respectiva manifestación o evento natural y el concepto de amenaza. El evento natural -terremoto, erupción, tsunami, deslizamiento, entre otros- es el resultado de procesos muchas veces ocultos tanto para la sociedad como para la geología. Se estudian desde el proceso que los originó y se determinan según parámetros físicos medibles -magnitud, intensidad, aceleración, entre otros-. La amenaza es un concepto que relaciona su origen, sus características físicas con el entorno socioeconómico. La amenaza trasciende el evento geológico, se podría decir que amenaza es el evento revestido de una visión antropocéntrica.

\section{Artículos e informes técnicos conceptuales}

El primer documento publicado en la RGAC vinculado al tema del desastre, es el escrito por Paniagua (1986), que corresponde con una nota técnica respecto a los volcanes activos de Costa Rica. Identifica los peligros volcánicos que cada volcán pueda generar, con comentarios acerca de los procesos que deben establecerse para aplicar conceptos de prevención y de mitigación con el fin de evitar consecuencias funestas como la ocurrida en 1985 en Colombia durante la erupción del volcán Nevado del Ruíz, para lo cual existen mapas de amenaza volcánica que establecen una zonificación específica para cada proceso amenazante que sea generado durante la actividad volcánica. El problema de esta nota técnica es que se confunde el uso de los conceptos de riesgo y de amenaza. 
Vergara (1987), hace referencia al "desastre natural" y lo define como "una situación que involucra pérdida de vidas, daño a las personas o la destrucción de la propiedad a una escala en que los servicios de emergencia normales son insuficientes". Igual que Paniagua (1986) utiliza desastre natural como sinónimo de evento natural pues los define como "fenómenos naturales cuya fuerza o duración puede ser catastrófica". Los conceptos de vulnerabilidad "el grado de pérdida de un elemento o grupo de elementos expuestos a un riesgo ocasionado por la ocurrencia de un fenómeno natural..." y riesgo específico como "el grado de pérdida debida a un desastre natural", no difieren en su definición. Luego separa los eventos de los desastres naturales cuando refiere "con la finalidad de definir las características de los eventos que pueden producir desastres naturales...". Aporta una relación de cálculo para el riesgo total que es igual a elementos en riesgo * peligro natural * vulnerabilidad.

También Vergara (1987) discute la zonificación de riesgos. Aporta dos posibilidades para construir la zonificación: 1. zonificación estática de riesgos, en la que considera los peligros en función de la gravedad acumulativa de daños, cualquiera que sea la frecuencia de su producción y una zonificación probabilística de riesgos, en la que se tiene en cuenta la frecuencia de los acontecimientos. ¿Cuál es la frecuencia de acontecimientos similares a Cartago 1910, o a eventos similares al huracán César, 1996, o la erupción del volcán Irazú, 1963?

Un aspecto del que se hace hincapié en la nota técnica escrita por Vergara (1987) es la frecuencia en que un evento específico se manifiesta, aspecto que apoya la idea de estimar una predicción para los eventos destructivos, vale considerar acá que los eventos geológicos no son susceptibles de predecirse por la complejidad de los procesos generadores. Además el autor advierte que debe existir una fluida comunicación entre la parte científica y las autoridades pertinentes, para que estas tomen las medidas necesarias ante una eventualidad, pero también con la población usando como vocero la prensa, sin caer en una exagerada consulta por parte de los medios que podría interrumpir el trabajo de los científicos en la recabación y análisis de los datos, o que se caiga en exageraciones a la hora de difundir la noticia.

Paniagua (1988) en una nota técnica presenta un resumen de la reunión de trabajo sobre prevención y previsión de desastres naturales en América Central. La nota técnica es importante porque rescata los pormenores de la reunión en la que se discutió la creación del Centro de Prevención de Desastres Naturales en América Central (CEPREDENAC). Cita los propósitos que en dicha reunión se identificaron para justificar la creación del CEPREDENAC que se pueden reunir en los siguientes: 1. Estudio e identificación de procesos naturales destructivos, identificación de las vulnerabilidades y el riesgo. 2. Intercambio de conocimientos mediante actividades formativas e informativas en la región centroamericana. 3. Creación de bases de datos regionales sobre eventos naturales destructivos. 4. Permanente formación de profesionales y técnicos; entre otros. Al leer los motivos justificantes, todos están relacionados con el desarrollo técnico científico de personal e instituciones de la región centroamericana, sin embargo, no se observa ningún motivo dirigido hacia el trabajo directo con comunidades vulnerables. Además, queda como error el hecho de llamar desastres naturales a los efectos derivados de los eventos naturales con la sociedad, tal como se comenta en el apartado teórico.

Paniagua (1995) define el desastre como alteraciones intensas en las personas, los bienes, los servicios y el "medio ambiente", causados por un suceso natural o por el ser humano. Sin embargo, en el contexto se sigue tratando los desastres como naturales. Además indica que los desastres deben ser entendidos como un importante problema de desarrollo. Genera una visión de los desastres como un ciclo que ya ha sido criticado líneas arriba.

\section{Amenaza en contexto urbano}

Dentro de contextos urbanos, se encuentran los trabajos de Salazar et al. (1992) y Mora (1993). Un análisis de las amenazas que eventualmente afectarían al acueducto Metropolitano es el escri- 
to por Salazar et al. (1992), quienes describen los procesos tectónicos y de geodinámica externa que afectarían obras civiles, tales como el acueducto metropolitano. Los autores refieren que debido al uso del suelo, las quebradas están ingresando en una nueva etapa de erosión, con el consiguiente peligro de generar avalanchas que rompan la tubería y que esto aumente el peligro hacia las áreas pobladas del valle del Río Grande de Orosi. Ubican procesos amenazantes de geodinámica externa con la infraestructura del área de estudio. Explican cuáles eventos de geodinámica externa podrían eventualmente afectar la infraestructura del área. Varias recomendaciones importantes van dirigidas a la preparación ante eventos de geodinámica externa.

El primer artículo que utiliza los conceptos de amenaza, vulnerabilidad y riesgo de manera cuantitativa es el escrito por Mora (1993) quien realiza un análisis de la amenaza, la vulnerabilidad y avanza en el cálculo del riesgo por lahares que transiten a lo largo del río Reventado, Cartago. Calcula caudales pico y procesos geológicos que se concatenan para aumentar las consecuencias de avenidas por el río Reventado. Propone una zonificación en función de la intensidad de la amenaza, hace un estimado de la vulnerabilidad de la infraestructura aledaña al área de influencia por lahares y asigna un valor estimado de vulnerabilidad para cada elemento de riesgo que el autor analizó. Además, calcula el valor, en colones, de las pérdidas esperadas en 1993, por efecto de un lahar que transite por el río Reventado.

\section{Eventos específicos: EI terremoto de Limón}

Una serie de artículos sobre temas asociados al desastre vienen contenidos en el número especial de la RGAC dedicado al Terremoto de Limón del 22 de abril de 1991, este evento sísmico significó la realización de observaciones multidisciplinarias para explicar el sismo y sus consecuencias ambientales. Sauter (1994) en un artículo evalúa los daños en estructuras civiles afectadas por el sismo, para lo cual genera un análisis a partir de criterios de la ingeniería estructural. Señala los efectos cosísmicos así como en algunos casos la calidad del material empleado en la construcción como responsables de la generación de daños en la infraestructura. Cortés et al. (1994) analizan el impacto ecológico en la zona entremareas, el sublitoral somero, ambientes severamente afectados a nivel de arrecifes.

Morales (1994) hace una evaluación económica de las pérdidas que alcanzó valores de 21 999,00 millones de colones US- $\$ 181,9$ millones) y plantea las medidas de mitigación pertinentes, que reconoce en la observación de la actividad sísmica, capacitación de comités locales, plan de vigilancia de cuencas, dragado y construcción de diques, actualización del código sísmico, reglamento de cimentaciones, atlas de amenazas naturales y vulnerabilidad.

Bermúdez (1994) todavía trata el tema como desastre natural y da a conocer las pérdidas sociales. Al abordar el tema de la información deja claro que fallas en este rubro ocurrieron por una inadecuada capacidad institucional de información, siendo así que la información fluyó de manera dispersa y no fue claro quién era el responsable de transmitirla. Una de las principales conclusiones para la época es la que señala que las autoridades competentes siempre dirigen su atención a los operativos de emergencia, en vez de dar énfasis a los aspectos de prevención. Además, realiza un estudio sobre el manejo institucional y la percepción de la población ante el sismo.

Mora y Peraldo (2011) construyen desde análisis de percepción de vecinos de varias comunidades de Pérez Zeledón el mapa de intensidades del terremoto de Buenavista del 3 de julio de 1983. Las representaciones sociales que recolectaron giran en torno al castigo divino, actividad volcánica o colapso de cavernas como los posibles motivos para que sismo se haya originado. Comparan los resultados con información de estudios sobre el terremoto y señalan que podría existir otra fuente sísmica para el terremoto de Buenavista, aparte de la que se conoce como falla Buenavista.

\section{Tsunamis}

Fernández (2002) analiza la amenaza que representan los tsunamis en las costas centroamericanas. Calcula que la estimación empírica de 
la amenaza por tsunamis es en la costa Pacífica desde Guatemala hasta Nicaragua y el Pacífico Central. En el Caribe, la estimación es el golfo de Honduras, Panamá y Costa Rica.

\section{Ordenamiento territorial}

Astorga (2001) aplica conceptos geológicos al ordenamiento territorial mediante los Índices de Fragilidad Ambiental (IFA) según un concepto integrador que el autor denomina geoaptitud de los terrenos, que considera un aporte de planificación. En el método se contemplan las amenazas naturales como un componente importante de ordenamiento territorial.

Esquivel (2004) escribe una nota técnica en la que hace referencia a la restricción del uso de la tierra en sectores con volcanismo activo, tal como el caso del Volcán Arenal. Hace un repaso de la información histórica de la actividad del V. Arenal y describe los factores formadores de vulnerabilidad en el área, tales como aumento de la oferta turística alrededor del edificio volcánico, aumento de la población, manejo de la información. Hace una propuesta de zonificación en los alrededores del volcán mediante cuatro zonas de restricción de uso. Esto es importante replicarlo para todos los volcanes activos del país, con el fin de trabajar en el tema de la gestión del riesgo en las comunidades cercanas al volcán. Es el caso del volcán Turrialba ha generado una actividad exhalativa profusa observada desde diferentes comunidades del cantón de Turrialba (Fig. 5).

Astorga (2013) en una nota técnica hace referencia a la definición del uso del suelo en las cercanías de fallas geológicas. Realiza una explicación sobre el protocolo técnico que fue publicado en el decreto ejecutivo 32967 del MINAE, que hace referencia al uso del suelo en los alrededores de fallas activas y potencialmente activas. El protocolo se basa en el establecimiento de zonas de seguridad especialmente enfocado a construcciones. Este protocolo tiene un importante impacto en el uso del suelo y en el ordenamiento territorial.

\section{Percepción del riesgo y de la amenaza}

En el tema de percepción del riesgo, Blunda (2010) hace un estudio social de la percepción de la población que vive en los alrededores del V. Poás, mediante una encuesta a personas de diferentes comunidades donde ellas aportan su apreciación respecto a una posible erupción del volcán. El estudio mostró que las personas encuestadas perciben el riesgo como bajo. Hay insuficiente divulgación de los planes de emergencia realizados para el área y la ausencia de un mecanismo capaz de actualizar dicho plan.

Mora (2014) escribe un artículo de actualidad en que refiere los antecedentes de la sentencia a los sismólogos que estudiaron el terremoto de L 'Aquila, Italia. Para entender el fallo judicial contra el grupo de expertos sismólogos, el autor hace referencia al concepto de predicción y a la falsa ciencia, además comenta el problema de la información sobre estos temas y la percepción de la población sobre el tema sísmico.

\section{DISCUSIÓN}

En los últimos años ha existido un boom en la investigación dirigida a medir el impacto de la producción científica en el desarrollo socioeconómico. Esto ha dado impulso a campos que se denominan cienciometría, a la que pertenece la bibliometría, todo con el fin de medir el uso de las publicaciones científicas y la producción de autores. En función de lo anterior, Pérez (2006) refiere que:

"En el último lustro el aparato gubernamental mexicano ha sido consistente en aplicar la máxima de que la aportación social de la ciencia y la tecnología no es tan clara y mucho menos directa. Como resultado, el apoyo federal a las actividades científicas ha disminuido de manera perceptible. En forma consecuente con esta actitud, cada vez es más evidente la delicadeza gubernamental por cuantificar el impacto de sus programas de ciencia y tecnología. En este contexto, la mayoría 


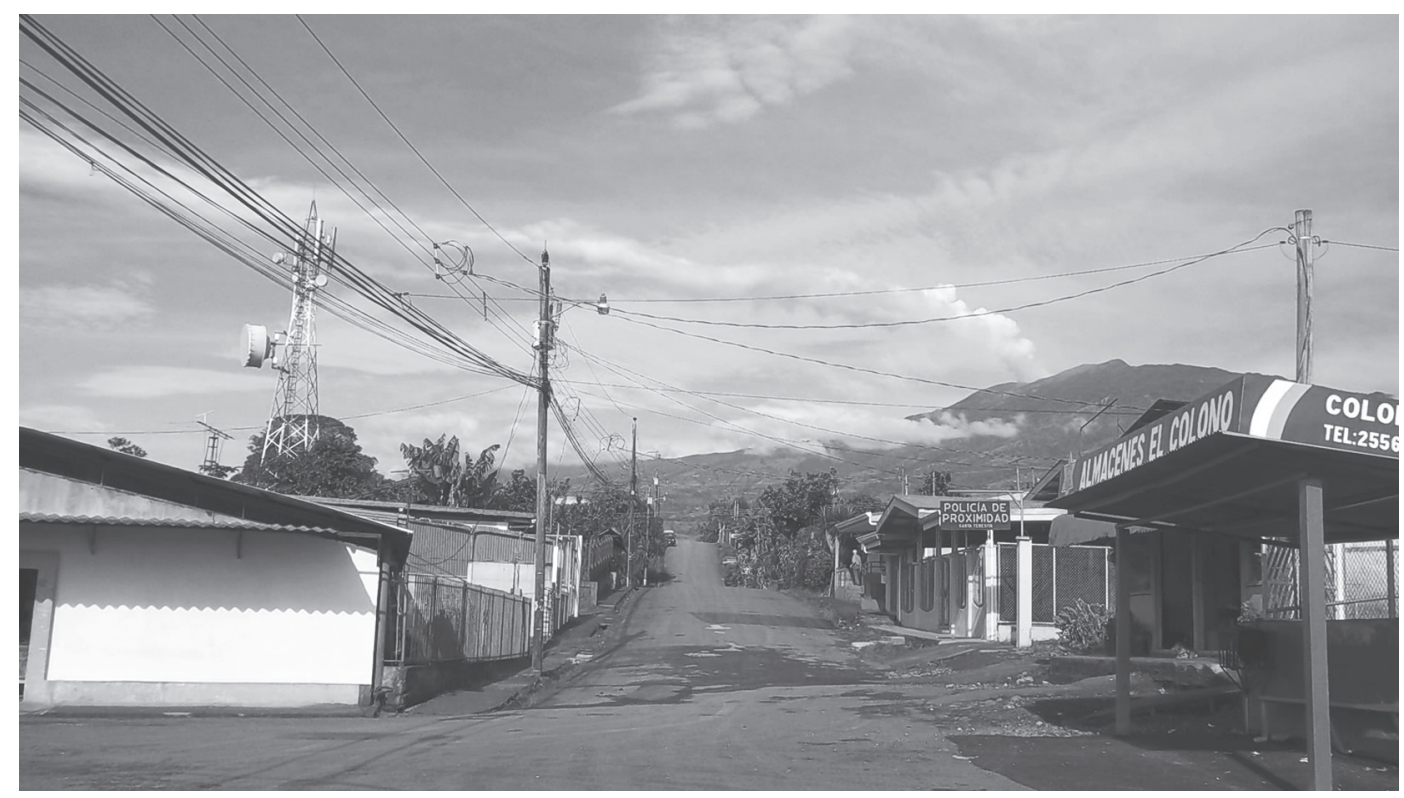

Fig. 5: Actividad exhalativa del volcán Turrialba vista desde Santa Teresita (antigua localidad de Lajas). Foto: G. Peraldo abril de 2013.

de las instituciones nacionales ha consolidado la cultura de la evaluación y del monitoreo de la investigación que desarrollan sus grupos de investigadores" (p. 29).

El análisis de las publicaciones científicas se ha perfeccionado, con el fin de responder al impacto en la sociedad, este impacto se evalúa según un criterio económico sobre cuánto invierte el Estado en investigación científica, y de demostrarse que dicha investigación es intrascendente, el Estado suprime los fondos. Los resultados de investigación pasan por dos momentos: en el primero se generan resultados sin aplicaciones aparentes, no hay una aplicación directa de los resultados, salvo que la ciencia se construya en función de lo meramente utilitario y no de curiosidad por estudiar un determinado fenómeno o cosa, y el segundo momento es la integración del resultado a una realidad concreta. Lo planteado por Pérez (2006) equivale a aplicar a la ciencia conceptos de trascendente o intrascendente. El problema de fondo, según lo anterior, radica en quién o quiénes definen qué investigación es intrascendente y cuál no, a cual grupo humano le parece que el tema es de importancia y mediante qué parámetros se llega a esa valoración. De lo contrario, se caería fácilmente en el negocio de producir "ciencia", según la visión de consumo del sistema económico que nos rige. Cabe también la posibilidad de orientar, de manera peligrosa, lo que el científico deba hacer, qué debe saber -aunque ese saber raye en un imposible- y cómo debe saberlo.

Lo anterior se aplica a la producción científica contenida en la RGAC. Concretamente, relacionado con el tema que nos tocó analizar, se pueden formular muchas preguntas. Una podría ser cuestionarse sobre la importancia de lo publicado para plantear argumentos que fueren contundentes para apoyar procesos de gestión del riesgo y de ordenamiento territorial. Ante esa interrogante es claro que los artículos que analizan procesos y eventos concretos aportan una gran cantidad de datos valiosos para ser traducidos al tema de la gestión del riesgo dentro del campo de la prevención. Por ejemplo, el estudio de un sistema neotectónico de fallas o los artículos que dan una explicación respecto a los mecanismos que generan erupciones en determinados volcanes de Costa 
Rica, llegan a cumplir un objetivo de gran realce, el cual es el estudio de las características del proceso amenazante. Por supuesto que una persona lega en los temas inherentes a la revista, no traducirá la información pertinente hacia su relación con la gestión del riesgo.

Queda claro, al revisar todos los números de la RGAC, que una buena cantidad de artículos y notas técnicas versan sobre el tema de la gestión del riesgo, pues se estudia el proceso (por ejemplo, un sistema de fallamiento neotectónico) que generará eventos peligrosos (como un sismo de gran magnitud). Sin embargo, en el tratamiento de las investigaciones, no queda del todo claro la conexión con la sociedad y la cultura; entonces, el problema de la desarticulación entre el resultado científico y su importancia para el desarrollo socioeconómico radica en la parcelación del conocimiento. Al leer un artículo que estudia un evento geológico tal como un sismo, debemos preguntarnos ¿cuál es la enseñanza concreta que debe generar en los lectores no especializados en el tema? En otras palabras, se nubla el vínculo entre lo científico y lo social.

Pero también el abordaje de la importancia social de un estudio puede tergiversar el sentido de los estudios sobre un determinado proceso. Tal como Mora (2014) analiza el caso de L'Aquila, Italia, respecto al grado de responsabilidad civil y penal de los sismólogos que no pudieron predecir el sismo que afectó la región. Acá se nota la ponderación de los estudios específicos, dirigidos hacia el saber cuándo y dónde ocurrirá el evento. Esto, como ya se ha discutido es ponderado por la visión que ha acompañado el objeto de estudio del desastre. Es claro que los jueces implicados en el sonado caso, están dentro del paradigma de desastre natural. Consideran, para comodidad del gobierno local, que las muertes fueron consecuencia de no tener una predicción. Si se aborda el tema desde la gestión del riesgo, entonces, la responsabilidad de las muertes adquiere un nuevo enjuiciado: el estado y el gobierno local, por no prever las condiciones de vulnerabilidad existentes en la localidad afectada.

No obstante, el juicio a los científicos italianos, abre en nuestro medio la discusión respecto a la predicción de eventos sísmicos y su impacto positivo o negativo en la sociedad. Abre el tema de qué, cuánto y cómo debe hacérsele llegar a la sociedad temas científicos sensibles. Existe investigación en temáticas importantes desde las ciencias geológicas para aspectos de seguridad nacional, sin embargo no se observa claramente el proceso de divulgación de los resultados hacia la población interesada. Es así que es común que se caiga en protagonismos, amarillismos informativos, inexactitudes de todo tipo durante la trasmisión de los aspectos científicos hacia el interlocutor lego en temas geológicos.

Para el caso de la divulgación de los artículos publicados por la RGAC, las palabras clave seleccionadas por los autores, a partir del año 2003, muestran pocas veces el tema de la gestión del riesgo como tal o sus elementos constitutivos básicos (vulnerabilidad, amenaza, riesgo). Estas palabras clave son importantes cuando se indexan los artículos dentro de una base de datos internacional, si no se agrega la palabra clave vinculante, tal como amenaza, cuando se estudia un evento sísmico, el artículo quedará invisible para estudios relativos a gestión del riesgo.

La revisión de los artículos contenidos en treinta años de la RGAC, evidencia que aspectos relacionados a la gestión del riesgo evolucionaron a partir del antiguo concepto de desastre natural y ciclo de los desastres (Paniagua, 1986, 1988, 1995; Vergara, 1987) hasta aplicar conceptos tales como amenaza, vulnerabilidad, riesgo (Salazar et al., 1992, Mora, 1993, Fernández 2002, Sauter, 1994, Morales, 1994) y temas colaterales como percepción (Blunda, 2010, Mora, 2014), o temas relacionados con el ordenamiento territorial (Astorga, 2001, Esquivel, 2004).

En cuanto a la amenaza, se debe repetir que la mayor parte de los artículos contenidos en la RGAC tienen relación directa con el tema de Gestión del Riesgo, pues los estudios geológicos básicos, así como los especializados en neotectónica, vulcanología, sismología y geotecnia responden a las necesidades de desarrollo de la sociedad. Es claro que la revista aporta mucha información relacionada a los procesos geológicos y a los eventos resultantes de dichos procesos, no obstante, por la naturaleza de la revista, se analiza el proceso natural desde la visión física de la geología. En casi todos los 
casos el estudio científico de un evento sísmico, o de un sistema de fallas, no muestra su relación directa con la gestión del riesgo. El vínculo de los resultados y análisis de las investigaciones, al ámbito de la gestión del riesgo, quedará en función de la experticia de cada lector.

\section{CONCLUSIONES Y RECOMENDACIONES}

El riesgo es una característica intrínseca de la transformación del ambiente, por tanto el estudio cuantitativo y cualitativo de dicha transformación propicia insumos para la prevención y el ordenamiento territorial.

La RGAC presenta gran cantidad de artículos y notas técnicas en temas tales como sismología, vulcanología, tectónica, neotectónica y suelos, entre otros. Sin embargo, esos artículos no tienen un indicador que les señale a personas legas en el tema, la relación de lo tratado con aspectos de gestión del riesgo.

Son escasos los artículos que de manera explícita se relacionan con algún concepto propio de gestión del riesgo (amenaza, vulnerabilidad, riesgo).

En los artículos y notas técnicas de la RGAC se observa desde mediados de la década de 1990 un cambio en el paradigma del concepto de desastre natural y el ciclo de los desastres hacia el paradigma de la gestión del riesgo.

El cambio en el paradigma sobre el desastre y su naturaleza, deja obsoletos los estudios de predicción y de pronóstico de procesos geológicos tectónicos, por cuanto para efectos de desarrollar la prevención son más eficaces los estudios para la construcción de escenarios de riesgo.

Como recomendación es importante que se llame la atención con un indicador en las palabras clave, cuando un artículo contiene información importante para la planificación territorial hacia la gestión del riesgo. Lo anterior es útil para que dichos artículos no queden desapercibidos en una búsqueda digital de información para gestión del riesgo.

Además, se recomienda un seguimiento de la utilidad práctica para la gestión del riesgo de los artículos científicos de la RGAC mediante el análisis de las fuentes de información geológica y su año de publicación usadas en documentos relativos con los planes reguladores cantonales, sobre todo de los cantones que han sido seriamente afectados por diversos procesos geológicos. De ese análisis podría evidenciarse un conocimiento limitado porque la información no habría tenido mayor difusión hacia los sectores encargados de la planificación del país. Esto confirmaría o no la necesidad de un interlocutor entre la RGAC y la comunidad no geóloga. Ese interlocutor debe tener dos voces: desde la Comisión Nacional de Prevención de Riesgos y Atención de Emergencias (CNE por sus siglas tradicionales) para la traducción de la información y el Colegio de Geólogos de Costa Rica, como el garante de que la investigación geológica reciente se respete e incorpore en los planes reguladores cantonales de manera constante y efectiva.

\section{REFERENCIAS BIBLIOGRÁFICAS}

ASTORGA, A. \& CAMPOS, L., 2001: El cartografiado de la geoaptitud de los terrenos.Rev. Geol. Amér. Central, 24:103-110.

BERMÚDEZ, M., 1994: El manejo institucional y percepción de la población en el Terremoto de Limón.- Rev. Geol. Amer. Central, vol. esp. Terremoto de Limón: 211-224.

CORTÉS, J., SOTO, R. \& JIMÉNEZ, C., 1994: Efectos ecológicos del terremoto de Limón.- Rev. Geol. Amer. Central, vol. esp. Terremoto de Limón: 187-192.

ESQUIVEL, L., 2004: Restricción del uso de la tierra en áreas bajo amenaza volcánica: el caso del Arenal.- Rev. Geol. Amér. Central, 30:203-211.

FERNÁNDEZ, M., 2002: Daños, efectos y amenaza de tsunami en América Central.- Rev. Geol. Amér. Central, 26: 71-83.

MORA, S., 1993: Análisis preliminar de la vulnerabilidad económica de la ciudad de Cartago, a causa de los efectos 
de una avalancha que transite por el río Reventado, Costa Rica.- Rev. Geol. Amér. Central, 15: 65-80.

MORALES, L.D., 1994: Daños causados por el terremoto de Limón: pérdidas y medidas de mitigación.- Rev. Geol. Amér. Central, vol. esp. Terremoto de Limón: 201-210.

PANIAGUA, S., 1986: Consideraciones sobre el riesgo volcánico en Costa Rica.- Rev. Geol. Amér. Central, 4: 89-92.

PANIAGUA, S., 1988: Reunión de trabajo sobre la prevención y previsión de desastres naturales en América Central.- Rev. Geol. Amér. Central, 8: 131-134.

PANIAGUA, S., 1995: Los desastres naturales y sus implicaciones en América Central.Rev. Geol. Amér. Central, 18: 107-112.

PERALDO, G. \& ACEVEDO, B., 2014: Entre los intersticios de la malla social: los desastres en el imaginario colectivo.- En: LÉRTORA, C. (coord.): Territorio, recursos naturales y ambiente: Hacia una historia comparada. Estudio a través de Argentina, México, Costa Rica, Haití, Paraguay, Uruguay y Venezuela.- FEPAI, Buenos Aires: 285-318.

PERALDO, G. \& MORA, M.,2009: Geología y geografía desde las microhistorias y la percepción.- En: VIALES, R., AMADOR, J.A.\& SOLANO F. (eds): Concepciones y Representaciones de la Naturaleza y la Ciencia en América Latina.- Univ. de Costa Rica, San José.

PERALDO, G. \& MORA, M., 2009: El miedo y su derivación en mitos sociales ante erupciones volcánicas y terremotos ejemplificados en América Latina entre los siglos XVI y XX.- En: LÉRTORA, C. (coord.): Geografía e Historia Natural: hacia una historia comparada. Estudio a través de Argentina, México, Costa Rica y Paraguay.- FEPAI, Buenos Aires: 347-394.

MORA, M. \& PERALDO, G., 2011: Análisis macrosísmico del terremoto de Buenavista de Pérez Zeledón, 3 de julio de 1983, (M =6,1).- Rev. Geol. Amér. Central, 44: 4170.

PÉREZ, M.A., 2006: Usos y abusos de la cienciometría.- www.cinvestav.mx/Portals/0/ SiteDocs/Sec_Difusion/.../6\%20usos.pdf [Consulta: 20 de julio de 2014].

SALAZAR, L. OBANDO, L. \& MORA, R., 1992: Acueducto metropolitano, tramo embalse El Llano - Río Navarro (Costa Rica): un sitio bajo amenaza.- Rev. Geol. Amér. Central, 14: 85-96.

SANTOS, M., 1990: Por una geografía nueva.257 págs. Espasa-Calpe, Madrid.

SAUTER, F., 1994: Evaluación de daños en puentes y otras estructuras civiles causados por el terremoto de Limón.- Rev. Geol. Amér. Central, vol. espec. Terremoto de Limón: 171-186.

\section{SERVICIO GEOLÓGICO MEXICANO,} ht tp://www.sgm.gob.mx/index. php?option $=$ com_content\&task=view\&id $=84 \&$ Itemid $=85$ [Consulta: 21 de julio de 2014].

VERGARA, A., 1987: Importancia de la prevención para el manejo de las emergencias causadas por desastres naturales.- Rev. Geol. Amér. Central, 7:171-181. 